\title{
Mechanical and environmental models for ceramic matrix composites
}

\author{
Hui Mei ${ }^{\mathrm{a}}$, Laifei Cheng, Litong Zhang, Yongdong Xu and Qingfeng Zeng \\ National Key Laboratory of Thermostructure Composite Materials, Northwestern Polytechnical University, Xi'an Shaanxi
} 710072, China

Received 30 June 2007; accepted 15 September 2007

\begin{abstract}
This paper outlines results from an experimental investigation on carbonaceous ceramic matrix composites (e.g., C/SiC) into mechanical behaviour under both monotonic tensile and step loadings, and into environmental behaviour exposed to oxidizing atmosphere containing oxygen. Complex damage phenomena like matrix microcracking, interficial debonding, fibre breakage and oxidation can occur in the serviced composites and result in progressive strain increase. These mechanisms can be modeled and formulated from the microscopic to macroscopic scales in order to predict damage development and final failure. The proposed models show good agreement with experiments and the ability to describe more complex damage procedure.
\end{abstract}

Key words: Ceramic-matrix composites; mechanical properties; environmental damage; modeling

\section{Introduction}

Current and future engines for next generation of aircraft and space vehicle need better materials to increase their thrust-to-weight ratio with increased safety and reliability while at the same time reducing the life cycle cost. To achieve these goals, novel or improved materials are required (Christin et al. [1]). Ceramic matrix composites (CMCs) are one class of materials that are receiving a great deal of attention in this regard. Modeling of the constitutive behavior and damage mechanisms of such materials is of particular importance due to low weight and high reliability requirements.

In the recent literature, two analytical approaches may be distinguished. The first one is a phenomenological approach based on thermodynamics of irreversible processes (e.g. see Zhandarov et al. [2]). Damage is introduced by means of scalar or tensorial internal variables. Model parameters are determined from macroscopic experiments. Phenomenological models are particularly suited for structural analysis. In the second approach, micromechanical models are used to describe elastic and damage behavior of the composites' constituents and interfaces (e.g. see Rospars et al. [3]). Model parameters are identified either by microscopic measurements or by inverse identification. Micromechanical models permit a better understanding of damage and can be used for optimization purposes.

The present paper focuses on the modeling damage development for carbonaceous ceramic matrix compos-

\footnotetext{
${ }^{a}$ Corresponding author: phdhuimei@yahoo.com
}

ites under mechanical, thermal, and chemical applied conditions. The related failure mechanisms and criteria are discussed. These models' parameters are not only derived from micromechanical analyses, also from macroscopic experiments, thus combining the advantages of phenomenological and micromechanical approaches.

\section{Modelling}

\subsection{Mechanical behavior}

Under tensile loading, as illustrated in Figure 1, CMCs present a linear elastic response below the matrix crack stress $\sigma_{m c}$ until the initiation and propagation of matrix micro-cracks and the partial reopening of thermal cracks. In a second stage, multiplication of matrix micro-cracks and the associated fibre/matrix debonding are propagating until matrix crack saturation. The matrix crack saturation is rapidly achieved (load transfer being poor) and the total failure occurred almost immediately after this point of saturation. For composites that present high strain to rupture, after matrix crack saturation, a progressive load transfer to the fibres which then fracture progressively occurs. Another net of cracks corresponding to multiple cracking of bundles can occur. These composites present a broad non-linear domain and higher stresses without any 'plateau-like' domain. The energy and hits of acoustic emission below matrix crack stress $\sigma_{m c}$ was pretty less, that above the proportional limit obviously became large, indicating that the onset of significant matrix cracking correlated closely to the proportional limit 


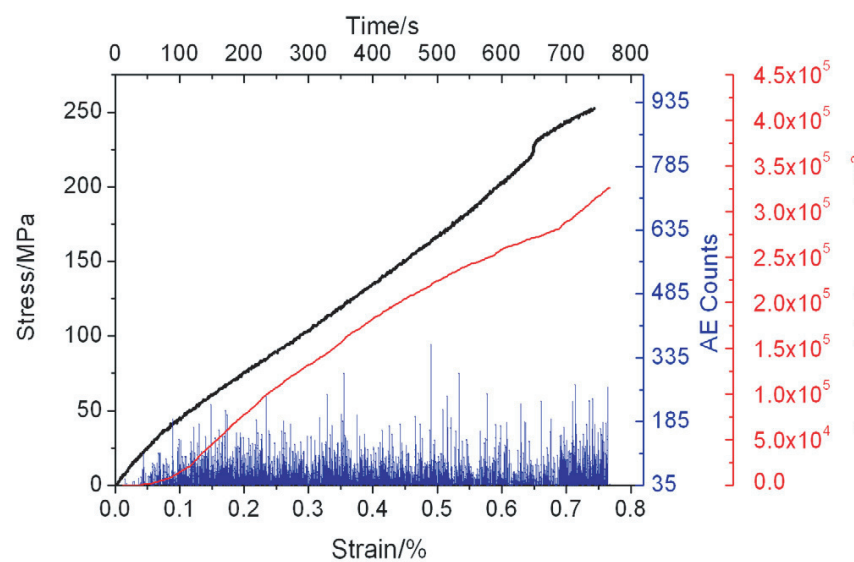

Fig. 1. Typical tensile stress-strain curve of a $2 \mathrm{D} \mathrm{C} / \mathrm{SiC} \mathrm{CMC}$ with real-time acoustic emission signals.

stress $\sigma_{m c}$. After an initiation period, the accumulated AE energy increased approximately parabolically with stress till final sudden and rapid zoom where a saturated matrix cracking state was believed to have been reached.

The tensile stress-strain relationship model can be obtained by an incremental loading-unloading-reloading sequence illustrated in Figure 2. The total strain, $\varepsilon$, is the sum of the inelastic strain and elastic strain:

$$
\varepsilon=\varepsilon_{i}+\varepsilon_{e}
$$

where $\varepsilon_{i}, \varepsilon_{e}$ denote the irreversible inelastic strain and reversible elastic strain upon loading.

A series of parallel and equally spaced transverse cracks are formed in the matrix once the applied stress is higher than the matrix cracking stress, $\sigma_{m c}$. The inelastic strain is simply the density of transverse matrix microcracks $\beta$ (i.e., number of cracks per $m$ ) multiplied by the crack opening displacement of each $\operatorname{crack} \delta$, as

$$
\varepsilon_{i}=\frac{\Delta L^{i n}}{L}=\frac{n \cdot U_{C O D}}{L}=\delta \cdot \beta
$$

where $n$ is the number of transverse cracks and $U_{C O D}$, crack opening displacement. Crack opening displacement of each crack $\delta$ can be simply estimate as (Begley MR et al. [4]),

$$
\delta=\frac{d V_{m}^{2} E_{m}^{2} \cos \varphi}{4 V_{f}^{2} \tau E_{f}\left(E_{f} V_{f}+E_{m} V_{m}\right)^{2}} \sigma_{A}^{2}
$$

where $\sigma_{A}$ is the applied stress, $\tau$ is the shear sliding stress of interface, $d$ is the fiber diameter, $\phi$ is braiding angles. $V_{m}, E_{m}, V_{f}$ and $E_{f}$ refer to volume fraction and Young's modulus of matrix and fiber.

As shown in Figure 2, the elastic strain in each steploading loop, related to the initial modulus $E_{0}$ and damage factor $D$, can be expressed as

$$
\varepsilon_{e}=\frac{\sigma_{A}}{E_{0}(1-D)}=\frac{\sigma_{A}}{\left(E_{f} V_{f}+E_{m} V_{m}\right)(1-D)} .
$$

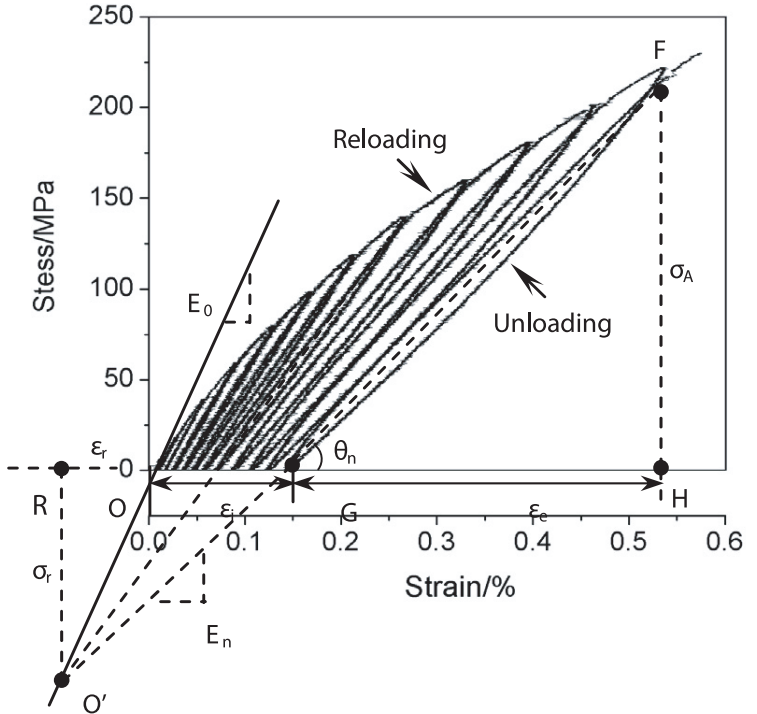

Fig. 2. Tensile stress-strain curve of a $2 \mathrm{D} \mathrm{C} / \mathrm{SiC} \mathrm{CMC}$ with interrupted unloading/reloading cycles.

Substituting equations (2), (3) and (4) into (1) gives a stress-strain relationship for CMCs:

$$
\begin{aligned}
\varepsilon=\frac{d \beta V_{m}^{2} E_{m}^{2} \cos \varphi}{4 V_{f}^{2} \tau E_{f}\left(E_{f} V_{f}+E_{m} V_{m}\right)^{2}} \sigma_{A}^{2} & \\
& +\frac{\sigma_{A}}{\left(E_{f} V_{f}+E_{m} V_{m}\right)(1-D)}
\end{aligned}
$$

On the other hand, the axial residual stress state at a given temperature and for a given composite specimen can be determined directly from the coordinates of that common intersection point by extrapolation of the compliance slopes of the secant modulus within each reloading/unloading loop. As shown in Figure 2, these compliance slopes happen to meet at a single point $O^{\prime}\left(\varepsilon_{r}, \sigma_{r}\right)$ localized in the compression domain, which can be approximatively calculated by using arbitrary two pairs of analogous triangles, e.g., $\Delta O^{\prime} R G \propto \Delta F H G$, as

$$
\frac{\sigma_{r}}{\sigma_{A}}=\frac{\varepsilon_{r}+\varepsilon_{i}}{\varepsilon_{e}}
$$

Thus, the thermal residual stress and strain can be calculated easily: $\varepsilon_{r}=-0.16 \%, \sigma_{r}=-134.85 \mathrm{MPa}$, which correspond well with the experimental results obtained by Camus et al. [5]. Hence, the monotonic tensile behavior of the ceramic matrix composite may be considered as damageable-elastic with respect to a fictitious thermalresidual-stress-free origin O' of the stress-strain axis.

\subsection{Environmental behavior}

In ceramic matrix composites, matrix cracks will serve as avenues for the ingress of the environmental atmosphere into the composite. In this analysis we are concerned with the effects of oxidizing environments on the reliability of a 
CMC at elevated temperatures. Neglecting the oxidation of the matrix, the following scenarios are considered for the interaction of the environment with the fiber coating:

I. it does not oxidize, but allows the diffusion of oxygen to the interior of the composite (e.g. an oxide fiber coating);

II. it oxidizes and forms solid oxide products (e.g. $\mathrm{SiC}$ );

III. it oxidizes and becomes fugitive (e.g. carbon).

Similarly, the following scenarios are considered for the interaction between the fibers and the environment:

I. the fibers do not oxidize (e.g. oxide fibers);

II. the fibers oxidize (e.g. non-oxide fibers).

As an example, we will focus the analysis to interactions of the type III and II for the fiber coating and the fibers, respectively. One of these typical $\mathrm{CMCs}$ is $\mathrm{C} / \mathrm{SiC}$ composites with carbonaceous fiber coatings (e.g., PyC interphase).

When the oxidizing environment ingresses into the composite a sequence of events is triggered starting first with the oxidation of the fibers and interphase. As a result, both the axial stress distribution in the fibers and their probability of failure will change. Consider the case when such the composite is subjected to a tensile stress $\sigma_{A}$, larger than the matrix cracking stress, $\sigma_{m c}$, the axial strain of the composite sample, according to the generalized Hooke's law, will increase with reduction both in the effective area of the reinforcing fibers from $A_{0}$ to $A(\mathrm{t})$ and in the volume average Young modulus as

$$
\begin{aligned}
\varepsilon^{*} & =\frac{\sigma_{A} A_{0}}{\left[\frac{A(t)}{A_{0}}\left(E_{f} V_{f}+E_{m} V_{m}\right)\right] A(t)} \\
& =\frac{\sigma_{A}}{\left(E_{f} V_{f}+E_{m} V_{m}\right)\left[1-2 \pi x /\left(a V_{f} \cos \varphi\right)\right]^{4}}
\end{aligned}
$$

where $x$ is the recession distance of carbon fibers from the surface into the interior of the composite, $\pi$ is the sinuosity coefficient of the gas diffusion path and $a$, the side length of square cross-section of the sample. In addition, in presence of the tensile stress the inelastic strain derived from the crack opening displacement due to fiber debonding, bridging and matrix cracking is also assumed to follow the equation (2). In equation (7), the oxidation of carbon phase is thought to be controlled by mixed diffusion of oxygen gas through the matrix microcracks. The recession distance $x$ can be properly developed, related to the exposure time $t$, as (Eckel et al. [6])

$$
x^{2}=K_{p} t=\left(4 D \frac{P}{\rho_{c} R T} \ln \frac{(1+\chi)\left(D_{k} / D\right)+1}{D_{k} / D+1}\right) t
$$

where $K_{p}$ is referred to as the parabolic rate constant. $\chi$ is the oxidant partial pressure, $P$ is the total pressure $(\mathrm{Pa})$, $\rho_{c}$ is the molar density of carbon $\left(\mathrm{mol} / \mathrm{m}^{3}\right), R$ is the gas constant $(\mathrm{J} / \mathrm{mol} \cdot \mathrm{K}), T$ is the absolute temperature $(\mathrm{K})$, $D_{k}$ and $D$ are Knudsen diffusion coefficient and Fick diffusion coefficient, respectively. The above equation (7) can

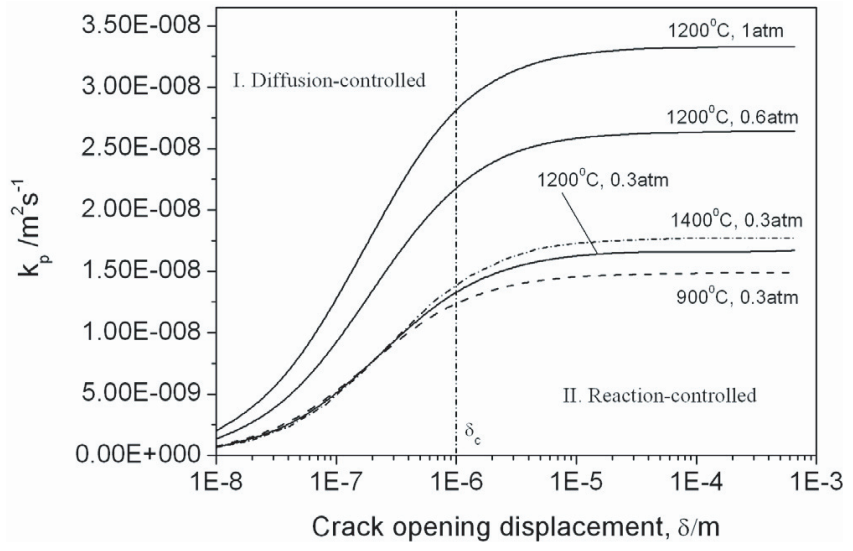

Fig. 3. Effect of crack opening displacement $\delta$ on recession rate $K_{p}$.

be rewritten as

$$
\begin{aligned}
\varepsilon^{*}=\frac{\sigma_{A}}{\left(E_{f} V_{f}+E_{m} V_{m}\right)\left[1-2 \pi \sqrt{k_{p} t} /\left(a V_{f} \cos \varphi\right)\right]^{4}} \\
+\frac{d \beta V_{m}^{2} E_{m}^{2} \cos \varphi}{4 V_{f}^{2} \tau E_{f}\left(E_{f} V_{f}+E_{m} V_{m}\right)^{2}} \sigma_{A}^{2} .
\end{aligned}
$$

Since the oxidation-assisted axis stress increase is a major contributing factor that can result in the increase of creep strain with the exposure time $t$, the portion of the inelastic strain is considered to be instantaneously completed at the beginning of loading and irrelevant to $t$. It is worthy to note that the established environmental model equation (9) may evolve into the mechanical model equation (5) when $t=0$ at the initial loading $(D=0)$.

Finally, it is interest to discuss the effect of characteristic dimension of the crack on the recession rate $K_{p}$ since the oxidation kinetics of the carbonaceous constitutes (e.g. PyC interphase or $\mathrm{C}$ fiber) is strongly dependent on the magnitude of $\delta$ in the oxidizing environments. Moreover, two common environmental parameters have significant influences on the dimensions of the crack: stress $\sigma_{A}$ and operating temperature $T$, the former open the crack according to equation (3) and the latter close the crack as a empirical formula found by Lamouroux et al. [7]. Therefore, the crack opening displacement in a nonreactive matrix can be obtained:

$$
\delta=\delta_{0}\left(1-\frac{T}{T_{0}}\right)+\frac{d V_{m}^{2} E_{m}^{2} \cos \varphi}{4 V_{f}^{2} \tau E_{f}\left(E_{f} V_{f}+E_{m} V_{m}\right)^{2}} \sigma_{A}^{2}
$$

where $T_{0}$ is the processing temperature and $\delta_{0}$, crack opening displacement at room temperature.

As shown in Figure 3, it is evident that temperature only weakly affects the magnitude and shape of the $K_{p}$ curve in the small $\delta\left(\leqslant \delta_{c}=1 \mu \mathrm{m}\right)$ regime. In the large $\delta$ regime, the speed of gas diffusion is greater than the reaction rate of the carbonaceous constitutes which procedure turns into the control-step and the oxidation kinetics change from the parabolic diffusion-controlled to the linear reaction-controlled regime. Increasing oxidant partial pressure is shown to accelerate the gas diffusion 
Table 1. Parameters and values used in calculation.

\begin{tabular}{llll}
\hline Parameter & Symbol & Value & Units \\
\hline Material & & & \\
Young's modulus of matrix & $E_{m}$ & 350 & $\mathrm{GPa}$ \\
Volume fraction of matrix & $V_{m}$ & 0.6 & \\
Young's modulus of fiber & $E_{f}$ & 230 & $\mathrm{GPa}$ \\
Volume fraction of fiber & $V_{f}$ & 0.4 & \\
Fiber diameter & $d$ & 7 & $\mathrm{um}$ \\
Side length of the cross section of sample & $a$ & 0.003 & $\mathrm{~m}$ \\
Braiding angle & $\phi$ & 22 & ${ }^{\circ}$ degree \\
Environmental & & & \\
Applied stress & $\sigma_{A}$ & Variable & $\mathrm{MPa}$ \\
Oxidant partial pressure & $\chi$ & Variable & $\%$ \\
Total pressure & $P$ & 101325 & $\mathrm{~Pa}$ \\
Molar density of carbon & $\rho_{c}$ & 150000 & $\mathrm{~mol} / \mathrm{m}^{3}$ \\
Gas constant & $R$ & 8.31441 & $\mathrm{~J} / \mathrm{mol} \cdot \mathrm{K}$ \\
Operating temperature $T$ & $T$ & 1573 & $\mathrm{~K}$ \\
Micromechanical & & & \\
Crack density & $\beta$ & 5300 & $\mathrm{~m}$ \\
Crack opening displacement & $\delta$ & Variable & $\mathrm{m}$ \\
Sliding stress of interface & $\tau$ & 3 & $\mathrm{MPa}$ \\
\hline
\end{tabular}

both in small and large $\delta$ regimes. More importantly, the higher oxygen pressure in the large $\delta$ regime seems to be fatal for the carbonaceous constitutes of the CMCs because the $K_{p}$ is enhanced dramatically with pressure.

\subsection{Implementation and simulation results}

Using the data presented in Table 1, the tensile stressstrain relationship and oxidation-assisted stress-rupture predictions were generated in Figures 4 and 5 by using the mechanical model equation (5) and environmental model equation (9) for a specific $2 \mathrm{D} \mathrm{C} / \mathrm{SiC}$ composite with a $\mathrm{PyC}$ interphase and a SiC-coating. Figure 6 plots the experimental variation and predicted time to failure (life) results for the tested $\mathrm{C} / \mathrm{SiC}$ composite samples as a function of the applied stress ratio $\left(\sigma_{A} / \sigma_{U T S}\right)$ by using the following failure criterion:

$$
R_{c}=\frac{A(t)}{A_{0}}=\frac{\sigma_{A}}{\sigma_{U T S}}
$$

where $R_{c}$ signifies the critical area reduction ratio, below which the progressively increasing tensile stress with decreasing $A(\mathrm{t})$ will reaches the UTS (ultimate tensile strength) of the materials leading to final rupture.

Despite slight deviation between theoretical predictions and experimental observations on the curves in Figures 4 and 6 , it is clear, without any attempt at fitting, that the established model not only matches the order of the observed results, but also follows the correct trend with increasing strain or stress. In the interest of simplicity but without losing generality, in contrast to the experimental observations, the oxidation-assisted stressrupture prediction illustrated in Figure 5 exhibits an intrinsic nature of the typical creep strain response of the CMCs in stressed and oxidizing environments (e.g. see Halbig et al. [8]).

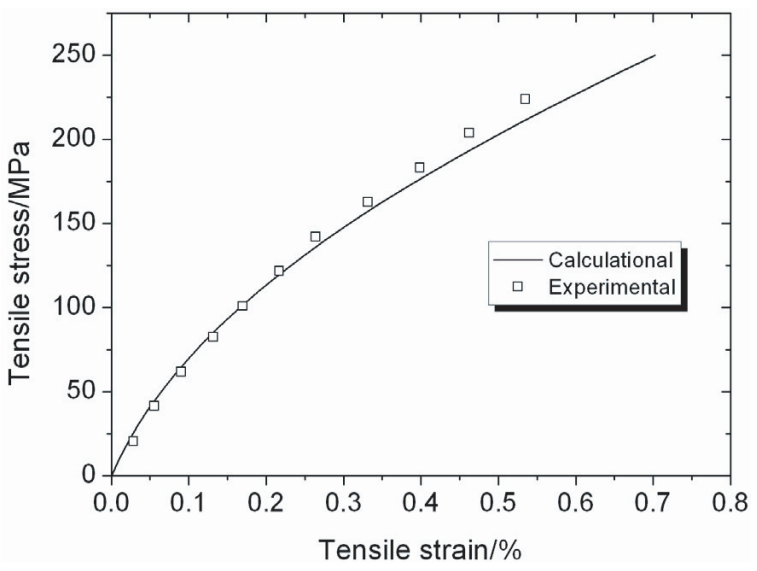

Fig. 4. Comparison of tensile stress-strain curves obtained by calculational and experimental methods.

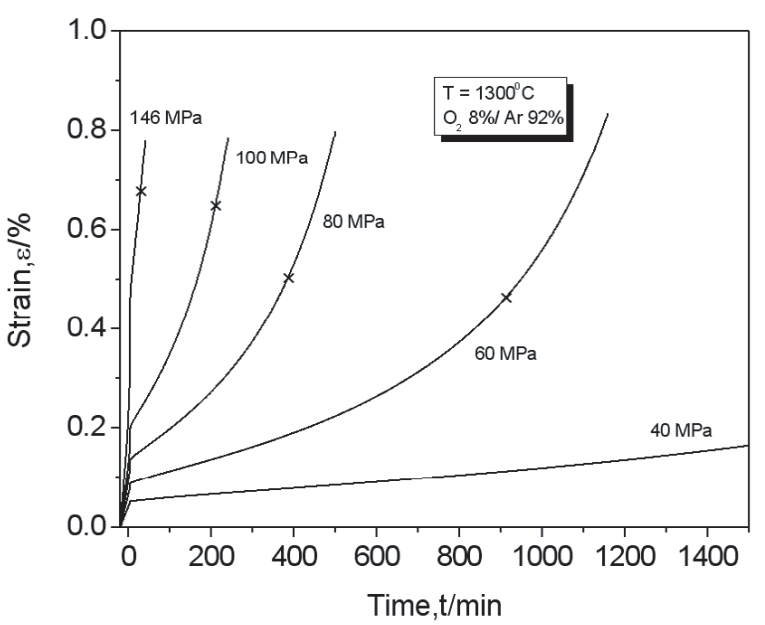

Fig. 5. Oxidation-assisted strain evolutions for a $\mathrm{C} / \mathrm{SiC}$ composite at $40,60,80,100$, and $146 \mathrm{MPa}$. 


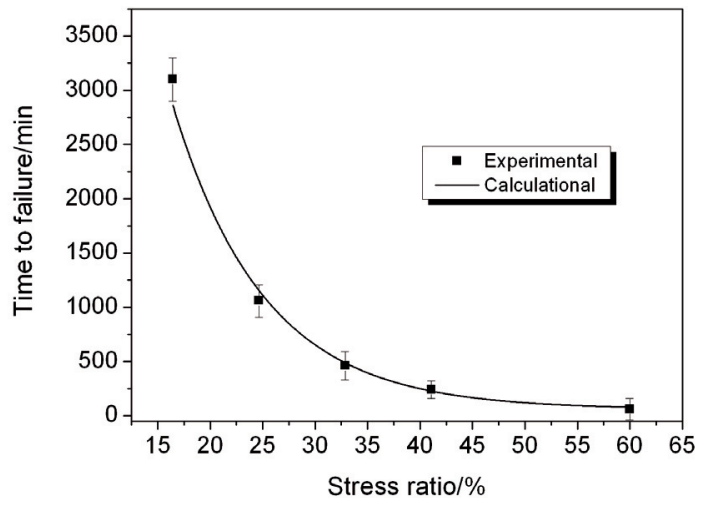

Fig. 6. Comparison of time to failure obtained by calculational and experimental methods.

\section{Conclusion}

Two theoretical models have been developed and correlated for the assessment of the intrinsic mechanical properties and the underlying environmental erosion behaviors of ceramic matrix composites based on the material, environmental, and micromechanical parameters. The modeled predictions are shown to not only match the order of the experimentally observed results, but also follow the correct trend for proper extrapolation. It will be necessary to further improve the environmental model to account for the oxidation of matrix when it is expected that matrix oxidation may lead to crack healing, altering in turn the kinetics of oxidation of carbonaceous constitutes.

Acknowledgements. The work is supported by the Natural Science Foundation of China (Contract No. 90405015) and National Young Elitists Foundation (Contract No. 50425208).
Appreciation is also extended to the Program for Changjiang Scholars and Innovative Research Team in university (PCSIRT).

\section{References}

1. F. Christin Design, Fabrication and Application of C/C, $\mathrm{C} / \mathrm{SiC}$ and $\mathrm{SiC} / \mathrm{SiC}$ Composites. In: Krenkel W, Naslain $\mathrm{R}$, Schneider H, editors. High temperature Ceramic Matrix Composites, Vol. 4. Wiley-VCH press, Weinheim (2001) p. 731

2. S. Zhandarov, E. Mader, Characterization of fiber/matrix interface strength: applicability of different tests, approaches and parameters. Comput. Sci. Technol. 65, 149$160(2005)$

3. C. Rospars, E. Le Dantec, F. Lecuyer, A micromechanical model for thermostructural composites. Comput. Sci. Technol. 60, 1095-1102 (2000)

4. M.R. Begley, B.N. Cox, R.M. McMeeking, Creep crack growth with small scale bridging in ceramic matrix composites. Acta Mater. 45, 2897-2909 (1997)

5. G. Camus, L. Guillaumat, S. Baste, Development of damage in a $2 D$ woven $C / S i C$ composite under mechanical loading: I. mechanical characterization. Comput. Sci. Technol. 56, 1363-1372 (1996)

6. A.J. Eckel, J.D. Cawley, T.A. Parthasarathy, Oxidation kinetics of a continuous carbon phase in a non reactive matrix. J. Am. Ceram. Soc. 78, 972-980 (1995)

7. F. Lamouroux, R. Naslain, Kinetics and mechanisms of oxidation of $2 D$ woven $C / S i C$ composites: Theoretical approach. J. Am. Ceram. Soc. 77, 2058-2068 (1994)

8. M.C. Halbig, A.J. Eckel. Degradation of continuous fiber ceramic matrix composites under constant load conditions, NASA TM-2000-210224 (July 2000) 